\title{
Atypical femoral fracture after receiving antiresorptive drugs in breast cancer patients with bone metastasis
}

\author{
Seiya Ota $^{1} \cdot$ Ryo Inoue $^{1} \cdot$ Takashi Shiozaki $^{1} \cdot$ Yuji Yamamoto $^{1} \cdot$ Naoki Hashimoto $^{2}$. \\ On Takeda $^{3} \cdot$ Kei Yoshikawa ${ }^{3} \cdot$ Junji Ito $^{3} \cdot$ Yasuyuki Ishibashi ${ }^{1}$
}

Received: 22 June 2016/ Accepted: 28 November 2016/Published online: 10 December 2016

(C) The Author(s) 2016. This article is published with open access at Springerlink.com

\begin{abstract}
Background Atypical femoral fracture (AFF) occurs with minor trauma in patients receiving antiresorptive drugs such as bisphosphonate and denosumab. We hypothesized that patients with bone metastasis who receive higher doses of antiresorptive drugs tend to experience AFF more frequently. This study aimed to investigate the prevalence rate of AFF in patients receiving antiresorptive drugs for bone metastasis of breast cancer.

Methods Based on the database from our hospital, patients with breast cancer between March and September 2014 were investigated. Thirty-two patients with bone metastasis who received higher doses of antiresorptive drugs were included for analysis and defined as the metastasis (M) group. For the control (C) group, 32 patients in the same period with breast cancer without bone metastasis who did not undergo antiresorptive drug therapy were included. We evaluated the localized periosteal thickening of the lateral cortex (beaking) and femoral neck-shaft angle in CT scout view, the periods from induction of antiresorptive drugs to the appearance of beaking, and the occurrence rate of complete fracture. The 2 groups were compared.
\end{abstract}

Seiya Ota

otaseiya012@gmail.com

1 Department of Orthopaedic Surgery, Hirosaki University Graduate School of Medicine, 5 Zaifu-cho,

Hirosaki 036-8562, Aomori, Japan

2 Department of Surgery, Aomori Prefectural Central Hospital, Aomori, Japan

3 Department of Orthopaedic Surgery, Aomori Prefectural Central Hospital, Aomori, Japan
Results Of the 64 limbs in 32 patients of the M group, 8 limbs in 6 patients showed beaking at the subtrochanteric area $(12.5 \%)$. After the occurrence of beaking, 5 limbs in 3 patients eventually had a complete fracture with minor trauma $(7.8 \%)$. On the other hand, no beaking was observed in the $\mathrm{C}$ group.

Conclusions The frequency of AFF in patients with breast cancer receiving bisphosphonate and/or denosumab for bone metastasis was high. More attention should be paid to the occurrence of AFF in these patients than osteoporotic patients.

Keywords Atypical femoral fracture - Bisphosphonate . Denosumab $\cdot$ Breast cancer $\cdot$ Beaking

\section{Introduction}

Atypical femoral fracture (AFF) occurs with minor trauma from the lesser trochanter to the supracondylar. This fracture configuration is transverse or short oblique, noncomminuted or minimally comminuted, and has focal or diffuse cortical thickening in the lateral cortex of the subtrochanteric or femoral shaft region. This localized periosteal or endosteal thickening of the lateral cortex is called "beaking" and is one of the major features of this fracture. Minor features are a generalized increase in cortical thickness of the femoral diaphysis, prodromal symptoms such as dull or aching pain in the groin or thigh, and delayed fracture healing [1].

AFFs have been reported as a potential complication of long-term bisphosphonate therapy for the treatment of osteoporosis [2]. There are many studies and case reports indicating that AFF could be more frequent in osteoporotic patients receiving antiresorptive drugs such as 
bisphosphonate and denosumab [3, 4]. Antiresorptive drugs reduce not only bone loss and risk of fractures in patients with osteoporosis [5], but also the frequency of skeletalrelated events such as pathologic bone fracture, spinal cord compression, and hypercalcemia due to cancer with bone metastasis [6-8]. The doses of bisphosphonates used for bone metastasis are up to 10 times greater than those for osteoporosis [9]. Similarly, the doses of denosumab used for metastasis are greater than those for osteoporosis. If antiresorptive drugs cause AFF, patients with bone metastasis who receive a much higher dose of those drugs than patients with osteoporosis might be at higher risk for AFF. However, there have been few reports that investigated the association between AFF and antiresorptive drugs in patients with bone metastasis.

Recently, we experienced several cases of AFF caused by minor trauma in patients with breast cancer undergoing antiresorptive drug treatment. In our hospital, computed tomography (CT) is performed in breast cancer patients to follow up for distant metastasis at the department of breast surgery. CT allows sequential image evaluation and early diagnosis of AFF in patients with breast cancer. We hypothesized that patients with bone metastasis who receive higher doses of antiresorptive drugs would tend to incur AFF at earlier phases and higher rates compared to those who do not receive antiresorptive drugs. The purpose of this study was to investigate the prevalence rate of AFF in patients receiving antiresorptive drugs for bone metastasis of breast cancer.

\section{Materials and methods}

\section{Patients}

Based on the database from the department of breast surgery in our hospital, patients with breast cancer between March and September 2014 were investigated. Of these, 53 female patients who were treated as bone metastasis were identified. An average age of these patients was 61.3 years (range 38-86 years). Detailed review of all medical records and imaging studies was performed retrospectively, and the height, weight, body mass index (BMI), past history, hormonal therapy, serum calcium and follow-up periods after induction of antiresorptive therapy from 2006 to 2015 were identified. The inclusion criteria were as follows: (1) use of intravenous pamidronic acid and/or zoledronic acid and/or subcutaneous denosumab as treatment for bone metastasis, and (2) clinical follow-up for at least 2 years after induction of high-dose antiresorptive drugs, and (3) CT scan taken from the neck level to proximal thigh level every 6 months for at least 2 years. Patients who had a history of osteoporosis or incomplete medical records were excluded. The remaining 32 patients were included for analysis.
These patients were defined as the Metastasis (M) group. For the control group, 32 patients who had breast cancer without bone metastasis, and did not undergo antiresorptive therapy in the same period were extracted. These patients were matched to the $\mathrm{M}$ group by sex, BMI, follow-up period and hormonal therapy, and defined as the control (C) group. The institutional review board of the Hirosaki University Graduate School of Medicine approved the protocol, and all patients and their families were informed that the data concerning their case would be submitted for publication. We obtained their consent.

\section{Imaging}

To determine the presence of AFF, all patients' CT images during the follow-up periods were evaluated by one investigator (S.O.) who knew the incidence of fractures. The checkpoint was the presence of localized periosteal or endosteal thickening of the lateral cortex at the subtrochanteric region (beaking) in CT scout view (Fig. 1). Beaking was defined as the presence of cortex extrusion. When it was difficult to judge beaking on the CT scout view, a senior orthopedic surgeon (R.I.) was consulted and a consensus was reached. After detection of beaking on $\mathrm{CT}$, the periods from induction of antiresorptive agents to the appearance of beaking, the occurrence rate of complete fracture, and the period from appearance of beaking to the occurrence of complete fracture were investigated. The femoral neck-shaft angle was measured in CT scout view. The femoral neck-shaft angle was defined as the angle formed by the intersection of a line down the centre of the femoral neck and a line through the centre of the femoral shaft. Bony union was defined as bridging of 3 of the 4 cortices on AP and lateral radiographs [10].

\section{Statistical analysis}

Data input and calculation were performed with the SPSS ver.12.0 J (SPSS Inc., Chicago, IL, USA). The comparison of age, height, body weight, BMI, follow-up period, and neck-shaft angle between the $\mathrm{M}$ group and $\mathrm{C}$ group was performed using the Student's $t$ test for parametric data and Mann-Whitney $U$ test for nonparametric data. Correlations of treatment duration of antiresorptive drugs and beaking or AFF were investigated with Spearman's correlation. In all analyses, $P$ values $<0.05$ were considered significant.

\section{Results}

Characteristics of the $\mathrm{M}$ and $\mathrm{C}$ groups are shown in Table 1 . The average age of the $M$ group was $59.2 \pm 11.6$ years, height was $152.8 \pm 6.7 \mathrm{~cm}$, weight was $54.2 \pm 8.8 \mathrm{~kg}$, BMI 
Fig. 1 Beaking of lateral cortex in scout view of CT. The presence of localized periosteal or endosteal thickening of the lateral cortex at the subtrochanteric region (beaking) in $\mathrm{CT}$ scout view
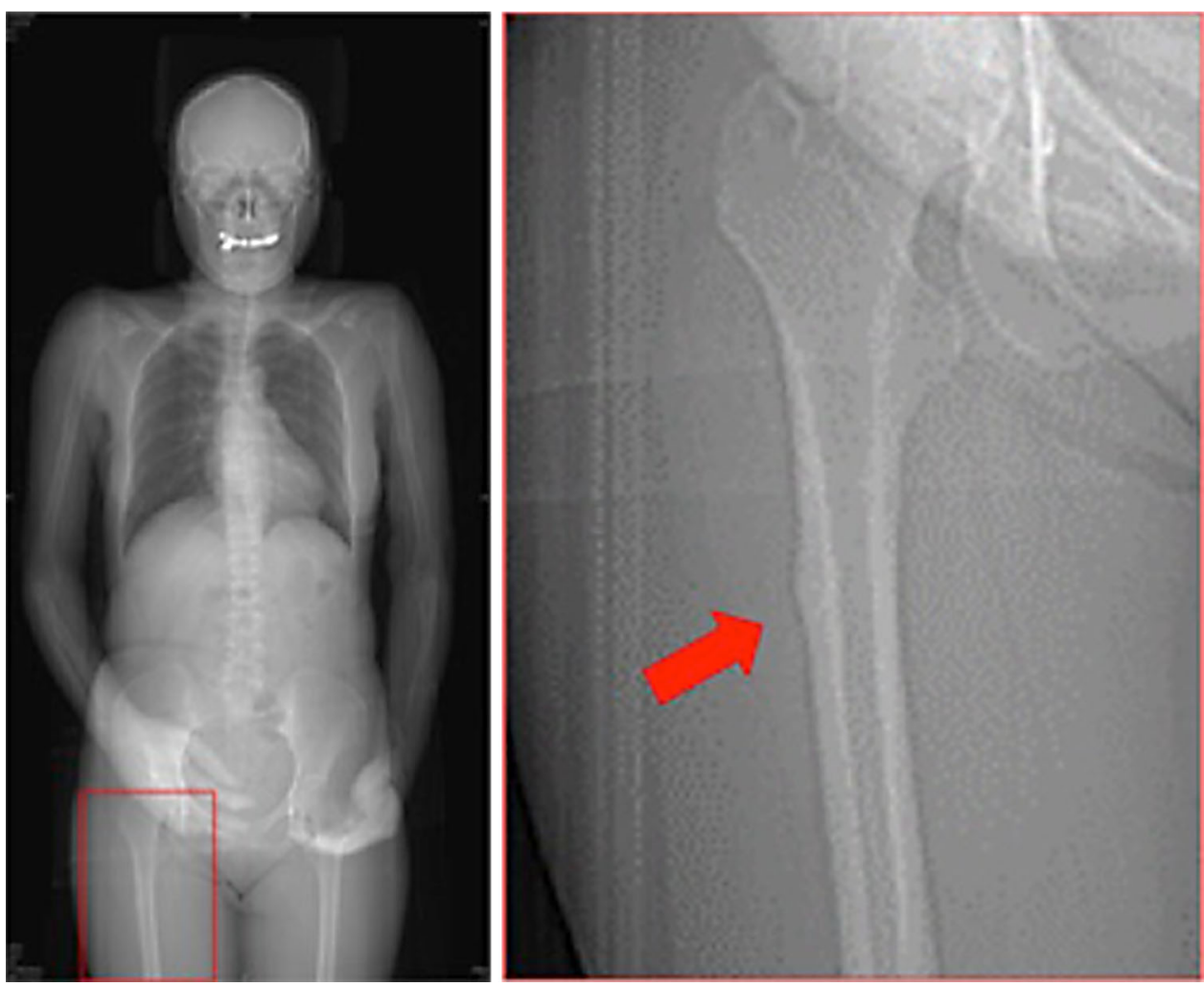

Table 1 Characteristics, follow-up period, hormonal therapy, and femoral neck-shaft angle of patients

\begin{tabular}{llll}
\hline & M group $(n=32)$ & C group $(n=32)$ & $p$ value \\
\hline Age (years) & $59.2 \pm 11.6$ & $61.9 \pm 13.4$ & 0.444 \\
Height $(\mathrm{cm})$ & $152.8 \pm 6.7$ & $154.4 \pm 5.8$ & 0.485 \\
Weight $(\mathrm{kg})$ & $54.2 \pm 8.8$ & $56.5 \pm 9.4$ & 0.452 \\
BMI $\left(\mathrm{kg} / \mathrm{m}^{2}\right)$ & $23.2 \pm 3.6$ & $23.7 \pm 3.6$ & 0.677 \\
Follow-up period (months) & $56.6 \pm 25.7$ & $53.5 \pm 27.4$ & 0.528 \\
Luminal type & $25(78.1 \%)$ & $26(75.0 \%)$ & 0.756 \\
HER2-positive type & $3(9.4 \%)$ & $3(9.4 \%)$ & 1.000 \\
Triple-negative type & 0 & $3(9.4 \%)$ & 0.119 \\
Unknown & $4(12.5 \%)$ & 0 & 0.057 \\
Chemotherapy & $20(62.5 \%)$ & $9(28.1 \%)$ & 0.006 \\
Endocrine therapy & $28(87.5 \%)$ & $28(87.5 \%)$ & 1.000 \\
Neck-shaft angle (right) & $140.7 \pm 6.4$ & $143.0 \pm 5.8$ & 0.056 \\
Neck-shaft angle (left) & $140.0 \pm 6.7$ & $140.7 \pm 6.6$ & 0.453 \\
\hline
\end{tabular}

The values of age, height, weight, BMI, follow-up period, and femoral neck-shaft angle are in mean \pm SD $P$ values below $0.05 *$ indicate significant level of difference between the $\mathrm{M}$ group and $\mathrm{C}$ group, using the Student's $t$ test for parametric data and Mann-Whitney $U$ test for nonparametric data was $23.2 \pm 3.6 \mathrm{~kg} / \mathrm{m}^{2}$, and follow-up period was $56.6 \pm 25.7$ months. On the other hand, the average age of the $\mathrm{C}$ group was $61.9 \pm 13.4$ years, height was $154.4 \pm 5.8 \mathrm{~cm}$, weight was $56.5 \pm 9.4 \mathrm{~kg}$, BMI was $23.7 \pm 3.6 \mathrm{~kg} / \mathrm{m}^{2}$, and follow-up period was $53.5 \pm 27.4$ months. There were no significant differences in age $(p=0.444)$, height $(p=0.485)$, body weight $(p=0.452)$, BMI $(p=0.677)$, and follow-up period
( $p=0.528$ ) between the 2 groups. One patient of the M group underwent pamidronic acid and zoledronic acid therapy, 12 underwent zoledronic acid only, 18 underwent zoledronic acid and denosumab, and 1 underwent denosumab only (Table 2). These antiresorptive drugs were not used concomitantly. Patients typically received $4 \mathrm{mg}$ zoledronic acid or $120 \mathrm{mg}$ denosumab every 3-4 weeks. The average of administration period of zoledronic acid was $43.5 \pm 28.0$ months. That of 
denosumab was $27.6 \pm 9.7$ months. There were many variations of past history in both groups, such as hypertension, diabetes, hyperlipidaemia, angina, cerebral haemorrhage and infarction, tuberculosis, cholecystitis, asthma, systemic lupus erythematosus, rheumatoid arthritis, Hashimoto's thyroiditis, Sjogren's syndrome, uterine myoma, endometriosis, ovarian

Table 2 Types of antiresorptive drugs in the M group

\begin{tabular}{lc}
\hline Type of antiresorptive drug & The number of patients \\
\hline Pamidronic acid, zoledronic acid & 1 \\
Zoledronic acid only & 12 \\
Zoledronic acid, denosumab & 18 \\
Denosumab only & 1 \\
Total & 32 \\
\hline
\end{tabular}

One patient of the $\mathrm{M}$ group underwent pamidronic acid and zoledronic acid therapy, 12 underwent zoledronic acid only, 18 underwent zoledronic acid and denosumab, and 1 underwent denosumab only

Table 3 The site for distant metastasis in subjects

\begin{tabular}{lll}
\hline & M group $(n=32)$ & C group $(n=32)$ \\
\hline Brain & $3(9.4 \%)$ & $1(3.1 \%)$ \\
Lung & $15(46.9 \%)$ & 0 \\
Liver & $15(46.9 \%)$ & $2(6.3 \%)$ \\
Stomach & $1(3.1 \%)$ & 0 \\
Bone & $32(100 \%)$ & 0 \\
No metastasis & 0 & $29(90.6 \%)$
\end{tabular}

The numbers of brain, lung, liver, stomach, bone and no metastasis are shown cyst, cervical cancer, gastric cancer, thyroid cancer, and malignant lymphoma. Two patients of the $\mathrm{M}$ group took oral prednisolone during the follow-up period. The sites for metastasis of $\mathrm{M}$ group were brain (3 patients), lung (15 patients), liver (15 patients), and stomach (1 patient). Those of $\mathrm{C}$ group were brain (1 patient) and liver ( 2 patients). The other 29 patients of $\mathrm{C}$ group did not have metastasis (Table 3 ).

Of the 64 limbs in 32 patients of the M group, 8 limbs in 6 patients showed beaking at the subtrochanteric area (12.5\%). On the other hand, in the $\mathrm{C}$ group, no beaking was observed. All 6 patients with beaking underwent bone scintigraphy to exclude bone metastasis. Five of six patients had no uptake of ${ }^{99 \mathrm{~m}} \mathrm{Tc}$ and one patient showed uptake in the subtrochanteric area. However, there were no findings of bone sclerosis and osteolysis suggestive of the metastasis on CT scan in this one patient. The period from induction of antiresorptive drugs to the occurrence of beaking was, on an average, 48.4 months (range 22-75 months). After the occurrence of beaking, 5 limbs in 3 patients eventually had a complete fracture with minor trauma (7.8\%) (Table 4). There were no patients with rheumatoid arthritis or received steroid treatment. Only 1 limb showed a complete fracture in the distal femoral shaft, and the site of this fracture was out of range of $\mathrm{CT}$ evaluation. All of these patients underwent surgery. The period from the presence of beaking to complete fracture was on, an average, 23.0 months (range 17-31 months). Only 1 patient with AFF (16.7\%) experienced hypocalcemia during the follow-up period, and 6 (23.1\%) patients without AFF experienced hypocalcemia in $\mathrm{M}$ group. The mean right femoral neck-shaft angles were $140.7^{\circ} \pm 6.4^{\circ}$ in the $\mathrm{M}$ group and $143.0^{\circ} \pm 5.8^{\circ}$ in the $\mathrm{C}$

Table 4 Characteristics of patients with AFF

\begin{tabular}{lllllllll}
\hline Patient & $\begin{array}{l}\text { Age } \\
\text { (years) }\end{array}$ & Side & $\begin{array}{l}\text { Metastasis (excluding } \\
\text { bone) }\end{array}$ & Drugs & $\begin{array}{l}\text { Duration } \\
\text { (months) }\end{array}$ & $\begin{array}{l}\text { Response to } \\
\text { therapy }\end{array}$ & $\begin{array}{l}\text { Beaking } \\
\text { (months) }\end{array}$ \\
\hline A & 50 & Right & Liver & Z & 74 & SD & 42 & $\begin{array}{l}\text { Fracture } \\
\text { (months) }\end{array}$ \\
B & 53 & Right & Liver & Z, D & 78 & PD & 62 & - \\
B & 53 & Left & Liver & Z, D & 78 & PD & 64 & - \\
C & 58 & Right & - & P, Z & 89 & SD & 41 & 68 \\
C & 58 & Left - & P, Z & 89 & SD & 46 & 77 \\
D & 64 & Right - & Z & 100 & PD & 35 & 52 \\
E & 68 & Right - & Z, D & 101 & PD & 75 & - \\
F & 73 & Right - & Z, D & 54 & SD & 22 & 39 \\
F & 73 & Left - & Z, D & 54 & SD & UNK \\
\hline
\end{tabular}

The period to beaking and complete fracture from induction of antiresorptive drug therapy is shown. Eight limbs in 6 patients showed beaking at the subtrochanteric area (12.5\%). The period from induction of antiresorptive drugs to the occurrence of beaking was, on average, 48.4 months. After the occurrence of beaking, 5 limbs in 3 patients eventually had a complete fracture from minor trauma (7.8\%). The period from the presence of beaking to a complete fracture was, on an average, 23.0 months. Patient F underwent AFF on the supracondylar of the left side, and this area was out of CT evaluation. Therefore, the occurrence time of beaking in the left femur was unknown. Bone metastasis's response to therapy was measured as progressive disease (enlargement of metastatic lesion on CT scan) or stable disease (no enlargement of metastatic lesion on CT scan)

$D$ denosumab, $P$ pamidronic acid, $Z$ zoledronic acid, $P D$ progressive disease, $S D$ stable disease, $U N K$ unknown 
group, and left were $140.0^{\circ} \pm 6.7^{\circ}$ in the $\mathrm{M}$ group and $140.7^{\circ} \pm 6.6^{\circ}$ in the $\mathrm{C}$ group (Table 1$)$. There were no significant differences in right $(p=0.056)$ and left $(p=0.453)$ between the 2 groups. Treatment duration significantly correlated with the presence of beaking ( $r=0.486, p=0.005$ ); on the other hand, it did not correlate with the occurrence of complete fracture $(r=0.285, p=0.114)$.

\section{Discussion}

This study showed that the occurrence rate of AFF in patients with breast cancer receiving antiresorptive therapy for bone metastasis was higher than that of those who did not receive antiresorptive drugs. Of those who received antiresorptive therapy, 8 limbs in 6 patients $(12.5 \%)$ showed beaking in the subtrochanteric area and 5 limbs in 3 patients $(7.8 \%)$ had a complete fracture. There are several studies on the frequency of AFF in patients using bisphosphonate for osteoporosis. Schilcher $\mathrm{J}$ et al. investigated 12,777 Swedish women using bisphosphonate for osteoporosis and reported 59 patients with AFF [11]. Black DM et al. reviewed 3 clinical trials and reported 12 of 14,195 patients with AFF [12]. As mentioned above, most previous reports about AFF were intended for the patients receiving lower doses of bisphosphonate typically used to treat osteoporosis. Two case series reported the frequency in patients with cancer. Puhaindran et al. investigated 327 patients with skeletal malignant involvement who received a minimum of 24 doses of intravenous bisphosphonates and reported 4 cases of AFF [13]. Chang et al. who investigated 62 patients with breast cancer or multiple myeloma with a femur fracture and prior intravenous bisphosphonate treatment for bone malignancy, reported 6 cases of AFF [14]. The frequency of AFF may have been higher (12.5\%) in our study because only breast cancer patients were subjects of investigation and all patients underwent CT scans to diagnose AFF.

The beaking appeared, on an average, at 48.4 months after antiresorptive therapy induction in patients with breast cancer and bone metastasis. On the other hand, no beaking was observed in those who did not undergo antiresorptive drugs in this study. There have been several reports on the period of fracture after bisphosphonate induction. Park-Wyllie et al. explored the association between bisphosphonate and fractures in a cohort of elderly women using bisphosphonate. They mentioned that among these elderly women, treatment with a bisphosphonate for more than 5 years was associated with an increased risk of subtrochanteric or femoral shaft fractures [15]. Also, there have been a few case reports for denosumab [16]. The mechanism of AFF and its association with antiresorptive drugs remains unknown. One hypothesis is that the chronic suppression of bone turnover by bisphosphonates may lead to an accumulation of microdamage in the bone, weakening and eventually leading to a fracture [3]. Therefore, there is an interval of several years from induction of antiresorptive therapy to the occurrence of AFF.

In this study, there was no significant association between the femoral neck-shaft angle and the occurrence of AFF. The cause of AFF is unknown and likely multifactorial. The biomechanical factor is one of the reasons for AFF. Hagen et al. mentioned that patients who presented with AFF had more varus proximal femoral geometry than those who did not sustain a fracture [17]. Oh et al. reported that of the 12 cases of low-energy femoral shaft fractures associated with bowing deformity, 6 cases were not treated with bisphosphonate at all [18]. They said that stress fractures associated with a femoral shaft bowing deformity should be recognized as another cause of AFF. In this study, there was no difference in the femoral neck-shaft angle between patients with AFF and without AFF in the M group. However, it is possible that our measurements overestimated or underestimated the actual varus in our patients because of limb rotation.

In regard to the complete fracture after beaking occurrence, this study showed that 3 of 5 patients with beaking definitely resulted in complete fracture (Fig. 2). There have been few reports about the prevalence rate of complete fracture after beaking occurrence due to AFF. However, in general, all incomplete AFF may progress to a complete fracture. Ha et al. mentioned that femoral insufficiency fractures after prolonged bisphosphonate therapy seldom healed spontaneously and most patients underwent surgery for fracture displacement or persistent pain [19]. Shane et al. reported that if there is no symptomatic and radiographic improvement after 2-3 months of conservative therapy, preventive nail fixation should be strongly considered as these patients may progress to a complete fracture [1].

Although the number of reports about the surgical treatment of AFF is increasing, it is unclear if a preventive surgery is needed or useful in incomplete fractures [20]. Several reports recommended conservative therapy that involves replacing bisphosphonate with teriparatide and limiting weight-bearing through the use of crutches or a walker. However, these conservative therapies have a significant negative impact on quality of life in these patients, and in a cancer patient, teriparatide is a contraindication. If a complete fracture occurs, the patient may require surgery and rehabilitation [21, 22]; therefore, the prevention of complete fracture is important. Routine X-rays should be undertaken to investigate any signs and symptoms associated with AFF. Preventive surgery may be effective to avoid complete fracture. Chang et al. retrospectively investigated 20 incomplete, non-displaced AFF with 
Fig. 2 Complete fracture of the bilateral femur by AFF.

a Complete fracture of the right femur and beaking on the contralateral side. b Post open reduction and internal fixation of right femur. c Complete fracture of the left femur by AFF. $\mathbf{d}$ Post open reduction and internal fixation of the left femur

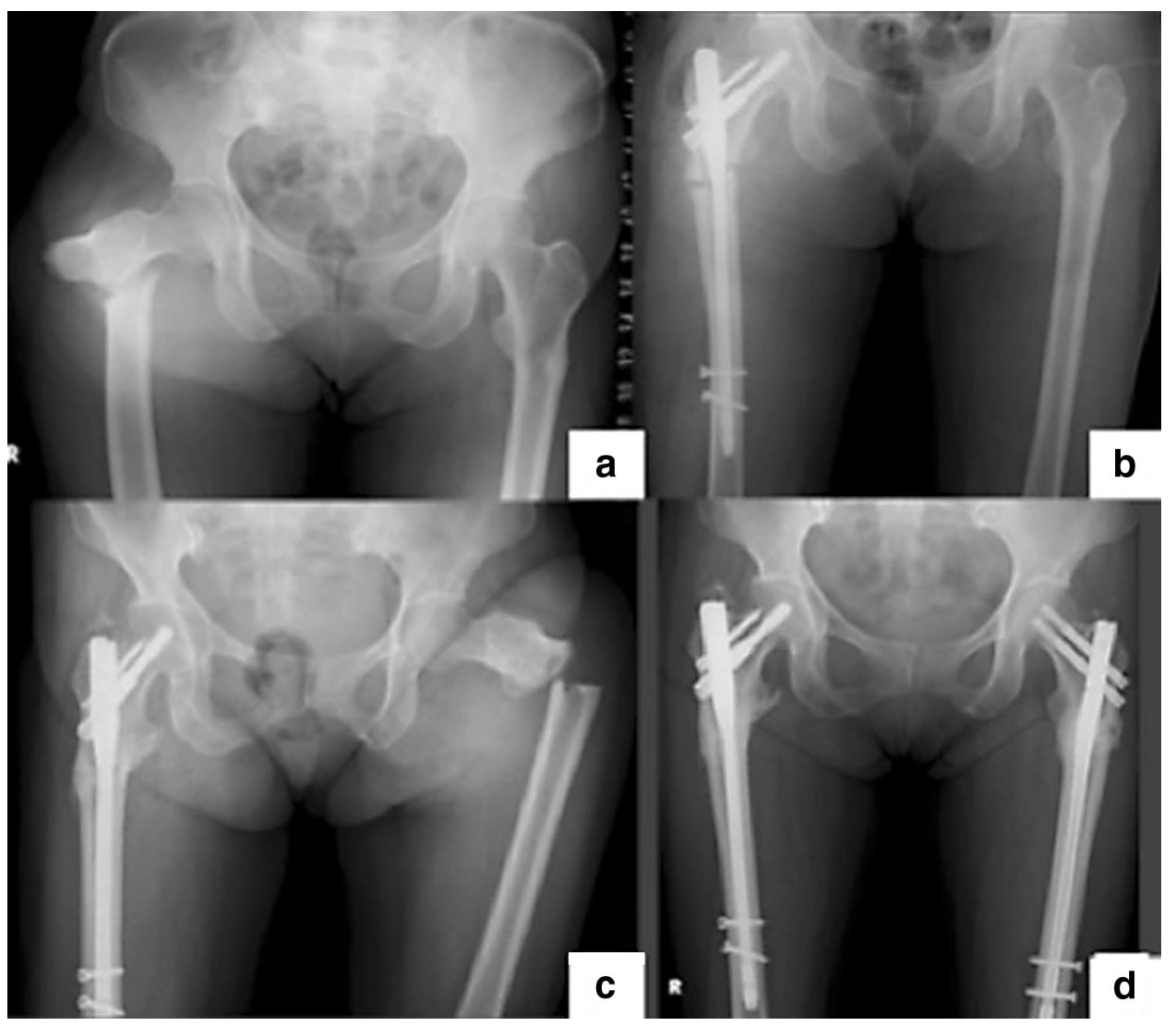

intramedullary nailing. They concluded that preventive fixation of AFF is recommended [14]. In this study, complete fracture after the appearance of beaking was seen in 5 of 9 limbs. Only 1 patient (1 limb) underwent preventive intramedullary nailing before the occurrence of complete fracture because transverse cortical fracture line inside beaking was detected, which was a possible precursor lesion of complete fracture. Of the complete fractures, 2 patients $(4 \mathrm{limbs})$ received intramedullary nailing and 1 patient (1 limb) received hemiarthroplasty. Bony union was observed 6-12 months after the operation. It is possible that preventive intramedullary nailing in patients with beaking is necessary.

This study had several limitations. First, as bone biopsy was not performed, histopathological examination was not studied. Second, bone mineral density measurements were not taken for the patients, which would have helped us to determine whether these patients had decreased bone density, which is a major risk factor for fragility fracture. Third, the range of CT was from the neck level to proximal thigh level; thus, not all of the femoral shaft was investigated.

In spite of above limitations, this study showed that the frequency of AFF in patients with breast cancer receiving bisphosphonate and/or denosumab for bone metastasis was higher than those who did not undergo antiresorptive drugs. Also, the rate of complete fracture after the appearance of beaking was high. Therefore, more attention should be paid to the occurrence of AFF in these patients than osteoporotic patients, and it is possible that preventive surgery for AFF before a complete fracture is necessary.

\section{Compliance with ethical standards}

Conflict of interest All authors have no conflicts of interest.

Open Access This article is distributed under the terms of the Creative Commons Attribution 4.0 International License (http://crea tivecommons.org/licenses/by/4.0/), which permits unrestricted use, distribution, and reproduction in any medium, provided you give appropriate credit to the original author(s) and the source, provide a link to the Creative Commons license, and indicate if changes were made.

\section{References}

1. Shane E, Burr D, Abrahamsen B, Adler RA, Brown TD, Cheung $\mathrm{AM}$, et al. Atypical subtrochanteric and diaphyseal femoral fractures: second report of a task force of the American society for bone and mineral research. J Bone Miner Res. 2014;29:1-23.

2. Shane E, Burr D, Ebeling PR, Abrahamsen B, Adler RA, Brown TD, et al. Atypical subtrochanteric and diaphyseal femoral fractures: report of a task force of the American Society for Bone and Mineral Research. J Bone Miner Res. 2010;25:2267-94.

3. Odvina CV, Zerwekh JE, Rao DS, Maalouf N, Gottschalk FA, Pak CY. Severely suppressed bone turnover: a potential 
complication of alendronate therapy. J Clin Endocrinol Metab. 2005;90:1294-301.

4. Drampalos E, Skarpas G, Barbounakis N, Michos I. Atypical femoral fractures bilaterally in a patient receiving denosumab. Acta Orthop. 2014;85:3-5.

5. Matsumoto T, Hagino H, Shiraki M, Fukunaga M, Nakano T, Takaoka K, et al. Effect of daily oral minodronate on vertebral fractures in Japanese postmenopausal women with established osteoporosis: a randomized placebo-controlled double-blind study. Osteoporos Int. 2009;20:1429-37.

6. Rosen LS, Gordon D, Kaminski M, Howell A, Belch A, Mackey $\mathrm{J}$, et al. Long-term efficacy and safety of zoledronic acid compared with pamidronate disodium in the treatment of skeletal complications in patients with advanced multiple myeloma or breast carcinoma: a randomized, double-blind, multicenter, comparative trial. Cancer. 2003;98:1735-44.

7. Rosen LS, Gordon D, Tchekmedyian S, Yanagihara R, Hirsh V, Krzakowski M, et al. Zoledronic acid versus placebo in the treatment of skeletal metastases in patients with lung cancer and other solid tumors: a phase III, double-blind, randomized trialthe Zoledronic Acid Lung Cancer and Other Solid Tumors Study Group. J Clin Oncol. 2003;21:3150-7.

8. Kohno N, Aogi K, Minami H, Nakamura S, Asaga T, Iino Y, et al. Zoledronic acid significantly reduces skeletal complications compared with placebo in Japanese women with bone metastases from breast cancer: a randomized, placebo-controlled trial. J Clin Oncol. 2005;23:3314-21.

9. Coleman RE, McCloskey EV. Bisphosphonates in oncology. Bone. 2011;49:71-6.

10. Frölke JP, Patka P. Definition and classification of fracture nonunions. Injury. 2007;38S:S19-22.

11. Schilcher J, Michaëlsson K, Aspenberg P. Bisphosphonate use and atypical fractures of the femoral shaft. $\mathrm{N}$ Engl $\mathrm{J}$ Med. 2011;364:1728-37.

12. Black DM, Kelly MP, Genant HK, Palermo L, Eastell R, BucciRechtweg C, et al. Bisphosphonates and fractures of the subtrochanteric or diaphyseal femur. $\mathrm{N}$ Engl $\mathrm{J}$ Med. 2010;362:1761-71.
13. Puhaindran ME, Farooki A, Steensma MR, Hameed M, Healey $\mathrm{JH}$, Boland PJ. Atypical subtrochanteric femoral fractures in patients with skeletal malignant involvement treated with intravenous bisphosphonates. J Bone Joint Surg Am. 2011;93:1235-42.

14. Chang ST, Tenforde AS, Grimsrud CD, O'Ryan FS, Gonzalez JR, Baer DM, et al. Atypical femur fractures among breast cancer and multiple myeloma patients receiving intravenous bisphosphonate therapy. Bone. 2012;51:524-7.

15. Park-Wyllie LY, Mamdani MM, Juurlink DN, Hawker GA, Gunraj N, Austin PC, et al. Bisphosphonate use and the risk of subtrochanteric or femoral shaft fractures in older women. JAMA. 2011;305:783-9.

16. Khow KS, Yong TY. Atypical femoral fracture in a patient treated with denosumab. J Bone Miner Metab. 2015;33:355-8.

17. Hagen JE, Miller AN, Ott SM, Gardner M, Morshed S, Jeray K, et al. Association of atypical femoral fractures with bisphosphonate use by patients with varus hip geometry. J Bone Joint Surg Am. 2014;96:1905-9.

18. Oh Y, Wakabayashi Y, Kurosa Y, Ishizuki M, Okawa A. Stress fracture of the bowed femoral shaft is another cause of atypical femoral fracture in elderly Japanese: a case series. J Orthop Sci. 2014;19:579-86.

19. Ha YC, Cho MR, Park KH, Kim SY, Koo KH. Is surgery necessary for femoral insufficiency fractures after long-term bisphosphonate therapy? Clin Orthop Relat Res. 2010;468:3393-8.

20. Oh CW, Oh JK, Park KC, Kim JW, Yoon YC. Prophylactic nailing of incomplete atypical femoral fractures. Sci World J. 2013.

21. Egol KA, Park JH, Rosenberg ZS, Peck V, Tejwani NC. Healing delayed but generally reliable after bisphosphonate-associated complete femur fractures treated with IM nails. Clin Orthop Relat Res. 2014;472:2728-34.

22. Grady MK, Watson JT, Cannada LK. Treatment of femoral fracture nonunion after long-term bisphosphonate use. Orthopedics. 2012;35:991-5. 